Res Pública Revista de Historia de las Ideas Políticas

ISSN: 1131-558X

http://dx.doi.org/10.5209/rpub.64253

\title{
Un instante de tiempo que lo transforma todo. Revolución marxista y construcción de situaciones en la Internacional Situacionista
}

\author{
Cristopher Morales Bonilla*
}

Recibido: 7 de mayo de 2019 / Aceptado: 31 de octubre de 2019

Resumen. Una de las mayores contribuciones de la I.S. fue el de transformar la concepción del cambio revolucionario. Partiendo de una superación del viejo concepto moderno de "revolución", los situacionistas entendieron que la transformación social solo era posible en el terreno de la vida cotidiana, es decir, en el conjunto de gestos y rutinas que conforman la vida de cada uno de los individuos y las comunidades.

Palabras clave: situación; revolución; Internacional Situacionista.; urbanismo unitario; deriva.

\section{[en] An Instant of Time that Transforms Everything. Marxist Revolution and Situation Building in the Situationist International}

\begin{abstract}
One of the main contributions made by the Situationist International was to transform the conception of revolutionary change. Taking the overcoming of the old modern concept of "revolution", the Situationists grasped that social transformation was only possible in the framework of everyday life, i.e., in the gestures and routines that conforms the life of every individuals and communities.
\end{abstract}

Keywords: situation; Revolution; Situationist International; Unitarian Urbanism; Dérive.

Sumario. 1. Las Thèses de Hambourg y la superación del arte. 2. El nuevo paradigma de la vida cotidiana. 3. La construcción de situaciones como práctica de transformación de la vida cotidiana. 4. La técnica de construcción de situaciones: la dérive. 5. Revolución marxista y construcción de situaciones. 6. Conclusiones: cómo hacer una transformación social con una situación construida. 7. Bibliografía

Cómo citar: Morales Bonilla, C. (2020). Un instante de tiempo que lo transforma todo. Revolución marxista y construcción de situaciones en la Internacional Situacionista. Res Pública. Revista de Historia de las Ideas Políticas, 23(1), 47-56.

Desde mitad del siglo XIX hasta nuestros días, el esquema del cambio social revolucionario parece haber permanecido fiel a sí mismo. Desde las luchas de los primeros movimientos sociales a finales del siglo XVIII $\mathrm{y}$ principios del siguiente $^{2}$, la transformación social parecía quedar reducida a un único concepto: revolución. Pese a la multitud de transformaciones políticas, sociales, económicas y culturales, el fantasma de la revolución parece haberse paseado por aquellos lugares y tiempos en los que se vislumbraba la posibilidad de un cambio radical. Ya fuera en las revoluciones burguesas de 1848, en la Comuna de París, en la Revolución de Octubre, en la Revolución española o en la cubana, parece haberse reproducido siempre la misma forma de pensar con respecto a la estructura del cambio social ${ }^{3}$.
Este esquema parece definirse por tres elementos. En primer lugar, una aceleración del tiempo, una concentración de todas las novedades, juntas en un mismo momento histórico. La revolución se entiende como un rayo que aparece en medio del fluir de la historia, como un trueno que surge de repente, con violencia, para cambiarlo todo en muy poco tiempo. Además, ese mismo concepto incluye la transformación radical de las condiciones de vida, de un estado de cosas determinado, ante el cual se oponen una serie de fuerzas históricas que entienden como insostenible a aquel. El concepto de revolución tiene dentro de sí un esquema simple pero radical: es necesario destruir un mundo para crear otro.

En tercer lugar, el concepto de revolución implica siempre una toma del poder: existe un lugar físico, de-

\footnotetext{
Universidad de La Laguna

cmoralbon@gmail.com

Sobre las condiciones materiales del nacimiento del movimiento obrero, cf. E.P. Thompson, La formación histórica de la clase obrera 1. Inglaterra: 1780-1832, Barcelona, Laia, 1977.

2 Para un estudio comparativo histórico-conceptual de varios procesos revolucionarios, cf., "Rethinking Revolutions: Integrating Origins, Processes, and Outcomes", en Comparative Studies of South Asia, Africa and the Middle East, Vol. 29, 1, 2009, pp. 18-32.
} 
limitable espacialmente, en el cual se ejerce el poder, desde el cual emanan las órdenes para mantener y conservar las condiciones materiales de vida. En todas las revoluciones modernas siempre ha existido un lugar que ocupar: la Bastilla, el Palacio de Invierno, etc ${ }^{3}$.

Sin embargo, pese a que cierta historiografía, probablemente influenciada por la hegemonía del marxismo en los estudios sobre dinámicas y transformaciones sociales, ha entendido que el concepto y la práctica revolucionaria han sido patrimonio de lo que en el siglo XIX empezó a llamarse "izquierda", lo cierto es que también su supuesto enemigo, la burguesía, ha sido igualmente revolucionaria ${ }^{4}$. Ya Marx en el Manifest entiende que la característica novedosa del, por entonces, naciente modo de producción capitalista es que es revolucionario en este sentido, es decir, libera unas fuerzas productivas tan poderosas que produce una destrucción total del mundo anterior (feudalismo) y una aceleración del tiempo por la cual todas las novedades históricas se concentran en un muy breve período de tiempo ${ }^{5}$.

A partir de la Segunda Guerra Mundial, los movimientos revolucionarios paradójicamente empiezan a abandonar la necesidad revolucionaria. Con la excepción de las revoluciones latinoamericanas, especialmente la cubana y el nacimiento del guevarismo, los movimientos políticos y sociales que, décadas antes trabajaban por hacer cumplir la necesidad revolucionaria, empiezan a transformar sus concepciones de la práctica política.

Dos factores contribuyen a esta nueva etapa: en el mundo capitalista occidental, el triunfo del keynesianismo y del Welfare State como una especie de final de la historia de las transformaciones revolucionarias. El Estado y el capitalismo consiguen crear sociedades en las que la contestación social parece casi desaparecer por completo. Por su parte, dentro del mundo del "socialismo real", la Unión Soviética se convierte en una sociedad administrada, en la cual la victoria de la Revolución de 1917 acaba por convertirse en una sociedad

El anarquismo, y todas sus variantes como el comunismo libertario o el anarcosindicalismo, serían una excepción a este elemento. Su intención es, más bien, la de crear un orden paralelo e independiente del poder hegemónico. Por eso, la conceptualización de la toma del poder se circunscribe a formulaciones marxistas de la revolución, es decir, a aquellas que entienden que el Estado cumple una función central en la configuración de las condiciones materiales de vida. Por otro lado, paradójicamente, movimientos contemporáneos como el 15M u Occuppy Wall Street sí que han vuelto a tener una forma espacial de entender el poder: en un caso, las plazas centrales de las grandes ciudades como lugares de reunión y encuentro; en el otro, la calle en la que se concentra la hegemonía financiera del planeta. Sobre el concepto de poder en el anarquismo, cf. W.O. Reichert, "Anarchism, Freedom, and Power", en Ethics, 79, 2, 1969, pp. 139-149; sobre la concepción espacial del poder en Occuppy Wall Street, cf., J. L. Hammond, "The Significance of Space in Occuppy Wall Street", en Interface: a journal for and about social movements, 5, 2, 2013, pp. 499-524.

$4 \quad$ En el período clásico de la producción teórica y la actividad práctica del movimiento obrero la burguesía es, efectivamente, el enemigo a batir. Sin embargo, en el siglo XX, y dentro del mundo del socialismo real, aparece la burocracia como otra de las clases sociales que se enfrenta a la posibilidad revolucionaria. Para un análisis de esta clase, cf. VV.AA., Socialisme ou Barbarie. Anthologie, La Bussière, Acratie, 2007.

5 Cf. K. Marx y F. Engels, Marx und Engels Werke (MEW). Vol. 4, Berlin, Dietz Verlag, 1977, pp. 462-474. Si no se específica lo contrario, todas las traducciones son propias. autoritaria en la que la contestación social tampoco parece ya posible, aunque por razones diferentes de las que ocurrían al otro lado del Telón de acero.

Precisamente, la década de los 50 es también el momento en el que toda una serie de grupos políticos minoritarios, mezclados con artistas de vanguardia y creadores culturales, empiezan a ensayar, de forma clara y decidida, experimentos sociales que intentan entender el cambio revolucionario fuera del esquema clásico de la práctica revolucionaria. Más en concreto, en Europa empiezan a aparecer una serie de pequeños grupos de activistas dentro del campo de la cultura que comienzan a entender las limitaciones de la concepción tradicional de la transformación social.

Dentro de esos grupos destaca la Internacional Letrista, que posteriormente se convertirá en la Internacional Situacionista (en adelante I.S.). Este grupo, heredero directo de las nuevas ideas que habían puesto en marcha el surrealismo, el dadaísmo y el futurismo, mezcladas con una interpretación de Marx filtrada por Karl Korsch y Gyorgy Lukács ${ }^{6}$, tiene como misión principal la de "transformar la vida", es decir, actualizar el concepto revolucionario de una forma que irá mucho más allá del esquema moderno ${ }^{7}$.

Por lo tanto, la Internacional Letrista, y la posterior I.S., suponen un momento decisivo en la historia de la transformación social del siglo XX, precisamente porque actualizan la necesidad de la transformación social, pero a partir de la crítica radical del comunismo soviético como una sociedad que, ya en esa década de los 50, había demostrado estar muy lejos de convertirse en alternativa al modo de producción capitalista. Por lo tanto, al declive de la concepción revolucionaria le corresponde su revitalización por parte de los situacionistas, retomando todas las investigaciones de las vanguardias europeas de principios del siglo XX.

Sin embargo, enfrentarse al modo de transformación revolucionaria de los situacionistas no está carente de todo tipo de problemas: ¿esta reconceptualización ha supuesto la creación de una estrategia más eficaz de transformación histórica? ¿Qué alcance ha tenido, y puede seguir teniendo, la forma de concebir la transformación social de la I.S., en un contexto en el cual dicha necesidad revolucionaria parece haber desaparecido? Dicho de otro modo: volver a la concepción de la transformación social de la I.S. tiene dos propósitos: por un lado, entender que el concepto común y tradicional de transformación social, el concepto de "revolución", sufre su crítica más profunda en la década de los 50 y 60 del siglo pasado; por otro lado, entender que, tal vez, esa misma reconceptualización de los situacionistas nos lleva a una forma de entender la transformación social que puede

Sobre la influencia de Korsch y Lukács en la I.S. y del modo en que entendían el marxismo, cf. S. Hastings-King, "L'Internationale Situationniste, Socialisme ou Barbarie, and the Crisis of the Marxist Imaginary", en Substance, 90, 1999, pp. 26-54.

En su texto fundacional, la I.S. ya establece como central la idea de "transformar la vida": "Pensamos que hay que cambiar el mundo. Queremos el cambio más liberador de la sociedad y de la vida en las que nos hallamos. Sabemos que este cambio es posible mediante las acciones adecuadas". VV.VV., Internationale Situationniste, Paris, Fayard, 1997, p. 689. 
servirnos hoy para entender qué acciones son importantes, y cuáles no, para la lucha política transformadora.

\section{Las Thèses de Hambourg y la superación del arte}

Dentro de la actividad de los situacionistas (1957-1970) se suelen distinguir dos momentos muy diferenciados: el primero, que enlaza con la actividad de la Internacional Letrista, se caracteriza básicamente por entender que es en el campo de la cultura en donde se libra la batalla decisiva de la transformación social. Así, los primeros números de la revista del grupo, Internationale Situationniste, están dedicados a dos cuestiones fundamentales: por un lado, a ejercer una crítica feroz al mundo cultural de la época, atacando ferozmente al surrealismo, a los antiguos letristas, a los movimientos de vanguardia y a los escritores consagrados del momento, etc ${ }^{8}$. Por otro lado, empiezan a concebir un proyecto revolucionario de transformación social completamente nuevo, acuñando términos tan importantes como deriva, situación construida o urbanismo unitario.

Sin embargo, en 1960, después de la quinta conferencia internacional del grupo celebrada en Goteborg, se produce un cambio radical en su dirección: se produce la expulsión de todos aquellos miembros que habían dedicado sus esfuerzos única y exclusivamente a la producción artística. La acusación es clara y muy significativa: el situacionismo se había convertido en una escuela artística, igual que le había ocurrido al surrealismo. Pocos días después, Guy Debord, Raoul Vaneigem y Attila Kotànyi se reúnen en un bar de Hamburgo para redactar un texto que constituiría el tiro de gracia a la actividad artística de la I.S, las Tesis de Hamburgo ${ }^{9}$. A pesar de que nunca fue publicado, son varias las referencias que mencionan este texto como el resumen de la crítica de la I.S. al mundo artístico.

Más allá de las luchas internas que hubo justo en ese momento dentro del grupo, lo importante es el significado de este giro, de este abandono: la sección dominada por Debord, por lo demás auténtico papa del situacionismo, había entendido que la lucha en el campo cultural había demostrado sus limitaciones como ámbito de la transformación más general. En su lugar, la I.S. se había convertido en una nueva tendencia artística, ya aceptada en el circuito del arte más vanguardista ${ }^{10}$. A partir de ese momento, la I.S. se dedicaría al análisis y la práctica más tradicionalmente revolucionarias en el sentido más

Sobre las críticas a surrealistas, letristas y diversos personajes del mundo cultural francés del momento, cf. Internationale Situationniste, op. cit., pp. 3-10 ss.

9 Sobre la historia de este texto, cf. Internationale Situationniste, op. cit., p. 703-704; VV.AA., Guy Debord. Un art de la guerre, Paris, Gallimard, 2013, p. 123 y ss; A. Hayes, "Three Situationists Walk into a Bar", en Axon, no 8, 2015 (http://www.axonjournal.com.au/ issue-8-1/three-situationists-walk-bar. Consultado por última vez el 15.04.2019). A pesar de que es uno de los pocos artículos en los que se estudia la importancia de Las tesis de Hamburgo, Hayes se equivoca al afirmar que el texto nunca fue escrito. La publicación de las notas manuscritas que llevan ese mismo título en Un art de la guerre demuestran lo contrario (p. 123).

10 Sobre el éxito de la I.S. dentro del mundo del arte de vanguardia, cf. Internationale Situationniste, op. cit., pp. 257-260. político, abogando por un comunismo de los consejos obreros y por la crítica del socialismo real como burocracia capitalista.

Sin embargo, visto en perspectiva, este giro estratégico significó una especie de salto hacia atrás. A pesar de su influencia en los acontecimientos de Mayo del 68, y de las esperanzas que ellos mismos vieron que se abrían a partir de ahí para la siguiente década de los 70, lo cierto es que en relación a la creación de nuevas formas de transformación social el abandono de su primera época "artística" supuso abandonar todos aquellos elementos más verdaderamente novedosos en relación a nuevas formas de contestación social.

No obstante, esta apuesta tenía poco recorrido en cuanto regresaba a una concepción revolucionaria ya ensayada a principios de los años 20 en los primeros experimentos de la Revolución Rusa. No hay que olvidar que ésta comenzó como una revolución de los soviets, tomando como modelo el comunismo de los consejos, los cuales eran el órgano principal de organización de la clase obrera. Obviamente, pronto la autogestión obrera pasó a convertirse en el centralismo democrático del Partido, quedando toda iniciativa del proletariado bajo su supervisión.

Por lo tanto, al volver a un modelo mucho más rígido y centralizado de transformación social los situacionistas cometieron dos errores estratégicos, que también son errores teóricos: por un lado, eliminar la experimentación en la vida cotidiana para regresar a la disciplina de la lucha del proletariado, para volver a reivindicar ese obrerismo mitologizado por el cual la única fuerza de contestación es el proletariado, especialmente su modelo clásico del trabajador de fábrica. El otro error que cometió fue el de aceptar ese dogma del marxismo por el cual es en el terreno de la contradicción entre fuerzas productivas y relaciones de producción en donde se libra la verdadera batalla final por la transformación social. Según este modelo, que con muchas variantes parece ser uno de los elementos compartidos por todas las formulaciones del marxismo, la cuestión de la supervivencia y su relación con el trabajo asalariado son el núcleo fundamental a partir del cual hay que explicar el resto de conflictos sociales. Tal y como Marx aprendió de los liberales clásicos como Adam Smith, la producción y el mercado son los elementos más importantes de toda vida social, los elementos a partir de los cuales es posible explicarla.

El gran problema de volver a este modelo por parte de la I.S. es que dicho obrerismo fue derrotado poco tiempo después del 68. Lejos de lo que ellos habían previsto $^{11}$, la centralidad de la lucha obrera empezó a verse derrotada por toda Europa. La década siguiente se repartió entre la huida hacia adelante de la lucha armada y la conversión de la radicalidad obrera en el reformismo del eurocomunismo o, directamente, en la defensa a ultranza de las dictaduras del socialismo real sudamericano, es decir, justo el modelo que los situacionistas habían jurado combatir.

\footnotetext{
1 Para la versión de la I.S. sobre el Mayo del 68, cf. Internationale Situationniste, op. cit., pp. 571-602; R. Viénet, Enragés: y situacionistas en el movimiento de las ocupaciones, Madrid, Castellote, 1978.
} 


\section{EI nuevo paradigma de la vida cotidiana}

Sin embargo, en ese primer período de actividad "artística" es en donde la I.S. llevó a cabo toda una serie de experimentos y técnicas de transformación que son las que hoy continúan teniendo significado para poder pensar nuevas formas de cambio social adaptadas a las condiciones materiales actuales. Si los situacionistas pudieron llevar a cabo todo ese conjunto de experimentos sociales es porque partieron de un paradigma muy diferente de cómo se debía entender la transformación. Fuertemente influenciados por Henri Lefebvre, el cual colaboró con la I.S. durante una época ${ }^{12}$, especialmente en el segundo volumen de su Critique de la vie quotidienne, los situacionistas adoptaron un nuevo marco en el que desarrollar la transformación social: la vida cotidiana.

Desde este punto de vista, la verdadera transformación social no se da en el ámbito del cambio de poder, de la toma del Estado y sus instituciones, como sí creía el marxismo-leninismo. Más bien, se trata de que la transformación social llegue a ser principalmente transformación de la cotidianeidad de los individuos:

Vayamos más allá y digamos que es en las cosas más familiares donde lo desconocido -no lo misterioso- se encuentra en la mayor riqueza, y que este rico contenido de vida todavía está más allá de nuestra conciencia vacía y tenebrosa, habitada como está por impostores, y atiborrada con las formas de la Razón Pura, con los mitos y su poesía ilusoria $^{13}$.

Por lo tanto, la perspectiva de la crítica cotidiana parte del punto de vista de Marx, en el cual la labor crítica tiene también un momento epistemológico anterior que no es otro que el instante en el que la crítica va más allá de lo aparente para llegar a la verdad. En el contexto de Marx, el descubrimiento de la verdad posee el momento revolucionario de desnaturalizar las formas sociales capitalistas, denunciando la explotación y la miseria allí donde solo se presenta trabajo asalariado y producción ${ }^{14}$. El paradigma de la vida cotidiana entiende que la transformación ha de llegar, principalmente, al conjunto de gestos y rutinas de la vida más íntima de los individuos y de las sociedades, a todo aquello que incluye no sólo las actividades normales como pueden ser el trabajo asalariado sino también al resto de acciones banales de los individuos. Dicho esquemáticamente: al día siguiente de la revolución no se puede seguir viviendo como si nada hubiera ocurrido ${ }^{15}$.

La importancia de este cambio de paradigma es enorme, no solo para la propia historia interna de la I.S., no solo para el conjunto de los grupos políticos más radicales que estuvieron en activo en los años 50-70, sino para el

12 Sobre la relación de Lefebvre con la I.S., cf. K. Ross, "Lefebvre on the Situationist: An Interview", en October, 79, 1997, pp. 69-83; H. Lefebvre, Les temps méprises, París, Stock, 1975, p. 109; A. Jappe, Guy Debord, Barcelona, Anagrama, 1998, pp. 89-90.

13 H. Lefebvre, Critique de la vie quotidienne, Vol. 1: Introduction, Paris, L'Arche, 1958, p. 145.

14 Es especialmente importante la conexión que hace Lefebvre del paradigma de la cotidianidad como un enfoque estrictamente marxiano. Cf. Critique de la vie quotidienne, op. cit., pp. $151 \mathrm{ss.}$

15 Cf. R. Vaneigem, Tratado del saber vivir para el uso de las jóvenes generaciones, Barcelona, Anagrama, 2008, pp. 221 ss. conjunto del devenir de la práctica política radical. No es exagerado afirmar que a partir de los análisis de Lefebvre, y de los modos y técnicas con las cuales los situacionistas intentaron concretar sus análisis, se produjo un cambio de paradigma absolutamente esencial dentro del movimiento revolucionario, el cual llega hasta nuestro presente. Este cambio es tan grande que podría resumirse diciendo que con la aparición de la cotidianeidad empezó a morir el paradigma moderno de la revolución.

Si la teoría hegemónica del cambio social, como ha sido el marxismo, ha tenido en la economía el pilar fundamental de su estudio y crítica, el nuevo paradigma de la vida cotidiana no solo incluye a éste sino que lo engloba en el conjunto de experiencias de un individuo dentro de unas condiciones materiales mucho más concretas.

No obstante, el paradigma de la cotidianeidad que abre Lefebvre no es exactamente el que toma la I.S.. La diferencia de enfoque radica en tomar lo cotidiano como objeto de conocimiento o como contenido de la vida liberada: “¿acaso la vida cotidiana actual es, a pesar de todo, un lugar de riquezas ocultas de donde puede partir una contestación generalizada, o es, por el contrario, un lugar de pobreza al que se ha de oponer la construcción de la vida verdadera?"16.

El problema que se plantea aquí es la diferencia que existe entre los surrealistas y los situacionistas en relación a cómo entender el cambio social. La primera perspectiva identifica lo cotidiano con lo maravillo en sentido surrealista, con lo que ya es un conjunto de vivencias plenas, las cuales estarían amenazadas por la necesidad de la subsistencia y del trabajo asalariado; por su parte, y este es el elemento crítico que introduce la I.S., la vida cotidiana se piensa como el lugar de la transformación, como la esfera en la que la actividad revolucionaria tiene que tener lugar y no como la esfera en la que dicha liberación ya ha tenido lugar.

Esta dialéctica es la que atravesará gran parte de los futuros movimientos de trasformación: o se establece una crítica de aquellas esferas en las que se parte de una cierta forma de identificación del proletariado con el trabajo como aquello propio y esencial, o se entiende que la posibilidad de lo cotidiano es la de la superación de la amenaza de la subsistencia. Por lo tanto, el paradigma de la cotidianeidad arroja una crítica social que es más radical en muchos aspectos de lo que era la crítica marxista. Ya no se trata de una crítica del modo de producción (capitalismo) y de la institución política (Estado). Se trata de entender cómo tienen que vivir los individuos y las comunidades en su día a día. Pero, además, es necesario comprender si esa cotidianeidad ya es un ámbito de liberación, antes de que entre la necesidad de la subsistencia, o si ésta lleva a la transformación revolucionaria aprovechando el desarrollo de los medios de producción.

La I.S. tomará la segunda perspectiva, entendiendo que la transformación no consiste en la creación de mundos oníricos, en la liberación de lo inconsciente, en extraer lo maravilloso de la experiencia de lo cotidiano, como si fuera el capitalismo y la explotación los que destruyeran la riqueza de lo cotidiano. Más bien, lo cotidiano es el lugar en el que es necesario aprovechar el desarrollo de las fuerzas

$\overline{16}$ Guy Debord, op. cit., p. 93. 
productivas para eliminar la necesidad del trabajo asalariado y de la explotación capitalista. No se trata de huir de lo cotidiano sino de transformarlo.

\section{La construcción de situaciones como práctica de transformación de la vida cotidiana}

Es en su primera época "artística" cuando la I.S. adopta el esquema de la cotidianeidad para llevar a cabo una serie de prácticas concretas con las cuales intervenir en los rituales, gestos y rutinas de los individuos en su vida diaria más concreta y material. La "construcción de situaciones" (construction des situations) es la práctica que los situacionistas fueron acuñando ya desde finales de su actividad en la Internacional Letrista hasta 1960. Ya desde el primero número de International Situationniste se da una definición exacta de lo que significa esta nueva forma de transformación radical: "Situación construida: momento de la vida construido concreta y deliberadamente para la organización colectiva de un ambiente unitario y de un juego de acontecimientos"17.

En esta definición la construcción de situaciones viene definida por dos elementos. En primer lugar, está atravesada por la organización colectiva de un "ambiente unitario". Con esta expresión hacen referencia a la dominación de todos los elementos que influyen en un contexto determinado para producir una experiencia determinada.

Para poder entender este primer elemento, es necesario tener en cuenta que la I.S. retoma el concepto clásico de Marx de "alienación", especialmente el desarrollo del par Entfremdung/Enttäuserung en los Manuskripte de $1844^{18}$ para reformularlo como "separación" (séparation). Con esta idea, los situacionistas entienden que la vida cotidiana está dividida en experiencias completamente diferenciadas las unas de las otras: el trabajo, el desplazamiento, el ocio o el sexo tienen todos sus momentos, sus geografías, pero especialmente sus tiempos y sus espacios. Tomando elementos de la crítica del urbanismo de Henri Lefebvre ${ }^{19}$, los situacionistas intentan superar esa alienación de la división de todos los momentos que conforman la vida cotidiana en las sociedades contemporáneas. Por lo tanto, la creación del "ambiente unitario" no es más que la forma en la que los situacionistas actualizan la crítica de Marx a la alienación en el trabajo asalariado, ampliada ahora a todos los aspectos de la cotidianidad.

Pero, además, para conseguir esa unidad de los gestos y rutinas de la vida cotidiana es necesario un control de aquellos elementos que son necesarios para construir una situación; dicho de otro modo, la situación parte de una dominación previa de aquellos elementos técnicos y materiales que influyen en la aparición de una situación buscada. Otra vez, el control que Marx entendía que los

Internationale Situationniste, op. cit., p. 13.

18 Cf. K. Marx y F. Engels, Gesamtausgabe (MEGA), I. Abteilung, Band 2, Berlin, Dietz Verlag, 2009, pp. 656 ss; Para la influencia de estos textos en la I.S., cf. A. Jappe, op. cit., pp. 45-57.

19 Cf. H. Lefebvre, La producción del espacio, Madrid, Capitán Swing, 2013. obreros tenían que ejercer en relación al producto de su trabajo es aquí generalizado al conjunto de toda la sociedad, a todo aquel elemento necesario para la construcción de una situación.

El segundo elemento de la definición de la construcción de situaciones es el "juego de acontecimientos" (jeu d'événements). Este concepto se refiere a la posibilidad de manipular, decidir, disponer libremente de todas las posibilidades a las que un ambiente determinado pudiera dar lugar. Se refiere a la manipulación de un medio material determinado para producir acontecimientos diferenciados, experiencias absolutamente únicas. Hay aquí una idea de producción que los situacionistas están usando, la cual, a diferencia de la aparición de lo "maravilloso" formulada por los surrealistas ${ }^{20}$, consiste en pensar que es necesario disponer de los medios materiales para producir la experiencia deseada. Existe aquí una voluntad de control y dominio del medio, y no un abandonarse ciegamente al azar y a las posibilidades no realizadas del ambiente.

\section{La técnica de construcción de situaciones: la dérive}

La construcción de situaciones incluye una serie de técnicas y prácticas a través de las cuales se llevan a cabo. Una de estas técnicas es la "deriva" (dérive). Esta es definida por la I.S. de la siguiente forma: "Modo de comportamiento experimental ligado a las condiciones de la sociedad urbana; técnica de paso ininterrumpido a través de ambientes diversos. Se usa también más específicamente para designar la duración de un ejercicio continuo de esta experiencia" ${ }^{21}$. La deriva, entendida de este modo, consiste en el vagabundeo por las calles de una ciudad determinada, dejándose llevar por la influencia de los ambientes encontrados, de las personas que se encuentran en el camino, etc ${ }^{22}$. A través de la deriva, el individuo es capaz de trazar nuevos caminos, nuevas rutas dentro de la ciudad, imitando al explorador que se adentra en la jungla desconocida, en territorio inexplorado. Con la deriva, el sujeto puede reapropiarse de su vida cotidiana al reapropiarse de su espacio vital, creando su propia ciudad al trazar sus propios caminos dentro de esa jungla de asfalto.

La metáfora del explorador que se adentra en un territorio desconocido con la intención de conquistarlo es aquí totalmente reveladora para el objetivo de la I.S. Sólo entendiendo las posibilidades del medio urbano como conjunto de ambientes con una capacidad clara de transformación del comportamiento es posible entender esta idea de la deriva y, a la vez, entender esa reinvención constante de la ciudad por parte de los situacionistas. Por lo tanto, es la aventura, la excitación ante lo desconocido, lo que se sitúa en la base de la deriva.

\footnotetext{
En cierto sentido, la aparición de lo maravilloso es un antecedente de la construcción de situaciones, aunque ésta última tiene un enfoque mucho más materialista en el sentido de la dominación de los elementos. Para entender el concepto surrealista de lo maravilloso, cf. A. Mahon, Surrealismo, Eros y politica, 1938-1968, Madrid, Alianza, 2009.

$21 \quad$ Internationale Situationniste, op. cit., p. 13.

22 D. Blanchard, La crisis de palabras, Madrid, Acuarela, 2007, pp. 37 ss.
} 
La deriva tiene principalmente dos ámbitos de desarrollo: "a) en el tiempo: constante, lúcida, influencial y, sobre todo, enormemente fugitiva; b) en el espacio: desinteresada, social y siempre apasionante"23. De este modo, la deriva incluye los mismos elementos que se pueden encontrar en la construcción de situaciones: la fugacidad, la pasión, el componente colectivo, etc. Sin embargo, estos dos ejes demuestran un elemento absolutamente fundamental: el carácter limitado de la acción, es decir, la conciencia de que cada gesto de transformación de la vida cotidiana incluye su propio límite. La deriva y la construcción de situaciones deben tener un principio y un final, están abocadas a su agotamiento desde el principio. Una vez cumplido su objetivo, la técnica deja de ser utilizada.

Uno de los ejemplos concretos, y frustrados, más conocidos de la deriva fue la que la I.S. intentó llevar a cabo en el museo Stedelijk de Amsterdam a principios de los años 60. Los situacionistas habían preparado un laberinto en varias salas con la intención de producir nuevas situaciones, al estilo del proyecto New Babylon de Constant $^{24}$. Cada sala tenía que estar destinada a la producción de una vivencia determinada, es decir, igual que ocurría con la división de barrios en el proyecto de Constant. Este laberinto tenía que ser completado con una deriva que se llevaría a cabo paralelamente por las calles de Amsterdam, estableciendo conexiones entre los componentes de la deriva y los miembros del laberinto.

Sin embargo, este proyecto nunca se llevó a cabo porque el museo pidió que el laberinto propuesto por los situacionistas tenía que ser supervisado por policías y bomberos para poder garantizar la seguridad del proyecto. La I.S. negó esta injerencia porque entendía que se introducía una instancia externa que modificaba la necesidad de que aparecieran nuevas situaciones, una nueva producción de ambientes. Una limitación exterior no podía más que imponer un límite a esa necesidad ${ }^{25}$.

El caso de la deriva del museo Stedelijk es importante por dos motivos: por un lado, porque fue la última situación construida de la I.S. Poco después, se llevaría a cabo la expulsión de los artistas de la I.S., abriéndose la segunda fase de su actividad marcada por el marxismo consejista; por otro lado, la deriva de Amsterdam reunió todos los elementos contradictorios de esa práctica, los cuales estallarían dentro de la organización de la I.S $\mathrm{S}^{26}$.

Internationale Situationniste, op. cit., pp. 51-55.

24 Para la influencia de New Babylon en la I.S, cf. M. Wigley, Constant's New Babylon: The Hyper-Architecture of Desire, Rotterdam, Witte de With, Center for Contemporary Art, 1998; H. Heynen, "New Babylon: The Antinomies of Utopia", en Assemblage, 29, 1996, pp. 2439.

25 Internationale Situationniste, op. cit., pp. 113-115; G. Debord, Correspondance. Vol. 1, Carta de Guy Debord a Willem Sandberg, 7 de marzo de 1960, Paris, Fayard, 1999, pp. 245-246.

26 Sobre la expectativa de la I.S. en la exposición en el museo Stedelijk destaca una carta de Debord a Constant: "No obstante, esta exhibición marca el paso (en el interior de la producción artística moderna) del objeto-mercancía, el cual es autosuficiente en sí mismo y cuya función es simplemente ser mirado, al objeto-proyecto, del cual la valorización es más compleja, desde que apela a una acción que debe ser tomada, una acción de un tipo superior que concierne a la totalidad de la vida". G. Debord, Correspondance. Vol. 1, Carta de Guy Debord a Constant, principios de junio de 1959, Paris, Fayard, 1999, pp. 182-184.
Una de las mayores contradicciones residía en el hecho de que la deriva perseguía un objetivo que, a su vez, era doble: por un lado, establecer el conocimiento del influjo del medio urbano en el comportamiento de sus habitantes; por otro lado, sin embargo, este conocimiento iba dirigido a la "recuperación" de ese mismo medio a través de la introducción de nuevas formas arquitectónicas, tales como las descritas en New Babylon. ¿Qué implicaba esta nueva configuración espacial? La introducción del azar dentro de la vida urbana: la rutina de la vida cotidiana tenía que ser superada y sustituida por la incertidumbre de la vida cotidiana liberada, por lo inesperado, por lo que no podía esperarse de antemano. La vida cotidiana tenía que convertirse en un conjunto de experiencias en las que el azar, lo inesperado, estuviera siempre presente como posibilidad. En definitiva: frente a la determinación de la vida cotidiana capitalista en la que todos los movimientos siguen un patrón determinado, los situacionistas buscaron, tal y como también hicieron los surrealistas, explotar el componente de aventura de la vida cotidiana.

Sin embargo, esta búsqueda del azar estaba relacionada con un conocimiento estricto de la influencia del medio urbano en el comportamiento, es decir, que existía una voluntad de determinar la influencia del medio social en la vida cotidiana. La contradicción se muestra aquí en que la liberación de la vida cotidiana no pasaba por una disminución del control medio urbano sobre los individuos, ni por un aumento de su libre movimiento e indeterminación. El azar, que sería el elemento en el que se materializaría una sociedad más libre, solo se podría conseguir a través de su producción. Tal y como quería representar New Babylon, la creación del azar solo se podía conseguir a través de un control exhaustivo de los usos y disposiciones de los espacios urbanos.

¿Cómo entender que la búsqueda del azar pueda ser controlada, racionalizada? En este ámbito, los surrealistas fueron mucho más coherentes: lo "maravilloso" sólo podía aparecerse, presentarse. El surrealista no tenía control de esa aparición. Simplemente daba cuenta de ella. Pero en los situacionistas el control del medio quería llegar al extremo de poder producir "situaciones" en las que se pudiera encontrar un elemento de azar, de libertad, de improvisación. En definitiva, ¿cómo podía ser que la situación, en lo que tenía de espontáneo, de azar, de experiencia liberada, fuera producida por la disposición determinada de ciertos elementos y técnicas disponibles en su momento? ¿Cómo producir aquello que tenía que ir más allá de la determinación?

Otra de las contradicciones, o limitaciones, de la deriva consiste en que tras la intención de producir un conocimiento exhaustivo de la ciudad la verdadera actividad se basaba en el paseo de cierto número de individuos que iban de bar en bar, dejándose llevar por el libre fluir de sus inclinaciones pasionales ${ }^{27}$. Desde esta perspectiva, la deriva parece quedar reducida a los juegos etílicos de ciertos individuos durante un corto período de tiempo y a través de un espacio determinado. Lo extraordinario es que, siendo esto cierto, los situa-

Cf. D. Blanchard, op. cit. 2007, pp. 37 ss. 
cionistas produjeron toda una serie de lo que llamaron "mapas psicogeográficos" (cartes psychogéographiques) que constituían verdaderos tratados en detalle de la influencia de todas las disposiciones de la organización urbana en el comportamiento de los individuos. Por lo tanto, la deriva, aún vista desde su perspectiva menos seria y más lúdica, fue ciertamente productiva para la construcción de una teoría y una práctica de una vida cotidiana liberada.

\section{Revolución marxista y construcción de situaciones}

La primera consecuencia que tiene la práctica experimental de la construcción de situaciones es que desplaza la importancia del concepto marxista de revolución, por lo menos en el contexto de la I.S.. La definición de la situación construida se sitúa en una perspectiva social en la que el trabajo asalariado ya ha sido abolido, aunque de forma abstracta, del mismo modo que Feuerbach había abolido la religión de una forma teórica. Dicho de otro modo: la I.S. se sitúa en esta primera época experimental en un contexto social que ya no es el del marxismo. A partir de ahí, y ya en su segunda época, los situacionistas se sitúan más allá del concepto formal de revolución, que la teoría de Marx había rescatado de la ideología burguesa, utilizándola entonces para la situación históricamente determinada de la clase obrera de los años 50-60 del siglo XX en los países desarrollados.

Al entender el contexto de la I.S. se puede entender el desplazamiento entre sus posiciones y las de Marx. En el período de formación de la teoría revolucionaria (segunda mitad del siglo XIX), la fe en el proyecto de recuperación de los medios de producción parecía inquebrantable. Sin embargo, todavía no se habían producido todas las revoluciones de carácter socialista que los situacionistas ya estaban denunciando bajo la etiqueta de "capitalismo de Estado" 28 , ni tampoco habían tenido lugar las traiciones de muchos partidos comunistas a la causa más puramente revolucionaria.

Por lo tanto, el contexto de la I.S. es el del diagnóstico de la revolución socialista como una práctica social no liberadora, carente de realidad en lo que tenía de emancipador en la teoría de Marx. La URSS, la China de Mao o la Cuba de Castro, pensaba la I.S., habían quedado reducidas a formas de "espectáculo integrado" 29 , no sólo porque en esas organizaciones sociales no se hubiera producido la reapropiación de los medios de producción por parte del proletariado (lo que se había producido era una apropiación de dichos medios por parte del Estado, identificándose éste con

\footnotetext{
8 Cf. Internationale Situationniste, op. cit. pp. 415 ss.

29 En Commentaires sur la société du spectacle, Debord matiza el concepto de espectáculo explicado en La société du spectacle. A las concepciones del "espectáculo difuso" (spectaculaire diffus) y del "espectáculo concentrado" (spectaculaire concentrée) le añade el "espectáculo integrado" (spectaculaire intégré), el cual estaría caracterizado por cinco rasgos: la innovación tecnológica incesante, la fusión de la economía y el Estado, el secreto generalizado, la falsedad sin respuesta y un presente perpetuo. Cf. G. Debord, Oeuvres, Paris, Gallimard, 2006, pp. 1597 ss.
}

el proletariado ${ }^{30}$ ) sino porque, allí donde sí se había producido, la vida cotidiana no había cambiado en lo esencial. La transformación de la propiedad de los medios de producción no había traído un cambio en las formas de vivir, en el modo en que el trabajador vivía su vida cotidiana.

Frente a la idea clásica de revolución, la idea de una construcción de situaciones transforma completamente el modo en el que se puede concebir una vida cotidiana liberada. Si en el concepto moderno de la transformación revolucionaria ha estado implícita la idea de una situación irreversible que cambia todas las condiciones de la existencia como consecuencia de un acontecimiento violento e inesperado que trastoca el devenir lineal de la historia, lo que aporta la idea de situación es la de un momento limitado en el tiempo que siempre está en permanente construcción.

El concepto de revolución implica la existencia de un estado de cosas que redime todas las injusticias pasadas y que, por este motivo, parece absolutamente definitivo. Es el momento final, el instante cumbre en el que la historia llegará a su fin cuando una de las clases, el proletariado, vencerá y establecerá la sociedad que la teoría marxista describe. Para los situacionistas, por el contrario, la situación construida es efímera, es siempre un momento en el que el individuo, y el grupo en el que se incluye, controla los efectos y las causas de su acción. Son instantes fugaces en los que aparece esa vida liberada. Esa fugacidad convierte a la construcción de situaciones en una actividad incesante, que no puede tener fin. La construcción de una vida liberada es la identificación entre la vida cotidiana y la construcción de situaciones.

Comparativamente, el concepto de situación de la I.S. implica un momento dialéctico en el que cada fragmento de vida liberada está siempre condenado a su final, a su límite, lo cual conlleva siempre una búsqueda incesante de nuevos ambientes, de nuevas aventuras, de nuevas situaciones. La historia no termina nunca porque nunca se dejan de producir situaciones. Si el concepto marxista de revolución tiene un momento dialéctico en su interior, en el desarrollo inmanente de la organización social, la construcción de situaciones posee este elemento no sólo en el proceso interno sino en su propio objetivo.

Aquí aparece la importancia del método dialéctico de Hegel: la construcción de situaciones es la aplicación práctica de la dialéctica hegeliana bajo una nueva forma: si en Hegel queda reducida a un marco idealista, según la crítica que le hace Marx; si en Marx la aplicación de la dialéctica todavía está dentro del esquema del concepto de revolución, en la I.S. aparece un tipo de "negación determinada", aplicado de un modo mucho más concreto a la producción de momentos diferenciados e identificables de nuevas formas de vida cotidiana.

\footnotetext{
Para uno de los mejores análisis de la apropiación privada de los medios de producción por parte del Estado en el socialismo real, cf. Ch. Laval y P. Dardot, Común. Ensayo sobre la revolución en el siglo XXI, Barcelona, Gedisa, 2015, pp. 69-108.
} 


\section{Conclusiones: cómo hacer una transformación social con una situación construida}

La principal crítica que se puede extraer del concepto y de la práctica de la construcción de situaciones es que es un modo de intervención en la realidad que está demasiado pegado al momento histórico en el que tuvo lugar. La influencia que en los años 50 tuvo la experiencia de los movimientos de vanguardia, tanto de dadaístas como, especialmente, de surrealistas produce que el modo de entender la transformación social quede demasiado atado al campo de la cultura. Por este motivo, la construcción de situaciones se sitúa en un plano diferente al que está el concepto moderno, y también marxista, de revolución.

Este plano diferente está caracterizado, a su vez, por la superación de dos coordenadas materiales que son fundamentales para el marxismo. En primer lugar, se sitúa por encima de la determinación del trabajo asalariado como actividad principal de la cotidianidad. Para la I.S., la construcción de situaciones no es que dé por efectiva el desplazamiento del trabajo, como si fuera un hecho social establecido, sino que parte de la posibilidad de que es necesario dedicar una cierta parte de la actividad de dicha cotidianidad a la experimentación, a la construcción de situaciones. Repitiendo un gesto que recuerda al de ciertos socialistas utópicos, la I.S. da por hecho aquello que tiene, todavía, que conseguir y demostrar: la superación del problema de la supervivencia, que es el segundo elemento que caracteriza el momento histórico de la construcción de situaciones.

En este sentido, la construcción de situaciones resulta una forma extraña de concebir la transformación social, en tanto que, de alguna forma, no es que dé por superado este problema sino que no lo pone en el centro de la actividad transformadora. Así, en comparación con la crítica de Marx, para la cual el método de la crítica de la economía política responde a la necesidad de poner en el centro el conjunto de problemas que se dan en la esfera de la producción, es decir, en el ámbito en el que los individuos buscan lo necesario para poder seguir viviendo, la construcción de situaciones parece situarse en un plano demasiado abstracto en relación a la posibilidad efectiva y real de la transformación social. No obstante, en cierto sentido, es la razón por la cual tras las Tesis de Hamburgo dan un giro radical hacia la perspectiva del comunismo consejista.

Sin embargo, es cierto que, desde un punto de vista más actual, el valor de las dos épocas de la I.S. parece matizarse un poco más, quedando la segunda etapa más "marxiana" en un segundo plano con respecto a la primera. El motivo de que este juicio haya cambiado después de varias décadas es, principalmente, lo mal que ha envejecido el marxismo, y más especialmente el comunismo consejista. Nuestro contexto más actual no es el de los años 60 del siglo pasado ${ }^{31}$, en el cual

\footnotetext{
Para entender ese contexto histórico-político de los años 60, cf. J. Varon, Bringing the War Home. The Weather Underground, the Red Army Faction, and Revolutionary Violence in the Sixties and Seventies, Berkeley/Los Angeles/London, University of California Press, 2004.
}

la posibilidad de una revolución social al estilo más clásico parecía no sólo posible sino probable, especialmente por el éxito de las revoluciones en países sudamericanos como Cuba o Nicaragua. En ese momento histórico, el Zeitgeist parecía prometer mucho más una revolución de los modos de producción que un éxito de la construcción de situaciones.

Parece obvio que nuestro contexto es muy diferente. Hoy, el marxismo como doctrina filosófico-política se enfrenta a un momento paradójico: por un lado, y a raíz de la crisis financiera de 2008, parece volver a vivir un cierto momento de revitalización, en el cual no sobran las lecturas que intentan actualizar a Marx o, incluso, volver a ciertas figuras del marxismo histórico como Lenin para ofrecer alternativas políticas y teóricas a problemas del presente.

Pero, a la vez, esta revitalización parece siempre tener que mirar hacia atrás, como si la respuesta a los problemas actuales consistiera en una reordenación, replanteamiento o resignificación de conceptos ya presentados en algún momento de la historiografía marxiana y marxista. Así, de lo que se trataría sería de investigar si estamos más cerca de las condiciones históricas descritas por el maoísmo, por el marxismo-leninismo, por la autonomía obrera italiana, o si estamos más cerca de lo analizado en Das Kapital o en los Grundrisse de Marx.

Hoy ya no disponemos de una clase obrera combativa que parezca encarnar la fuerza negativa de la historia, tal como podía pasar en el contexto de la I.S. ${ }^{32}$; tampoco disponemos de un movimiento estudiantil que pudiera parecer estar tomando el testigo del proletariado, como también podía parecer en los años 60 . Nuestro contexto es, por encima de todo, el de la derrota de los movimientos emancipatorios de aquella época. Nuestro marco general es el de la necesidad de volver a pensar cómo se cambian las sociedades, por qué se transforman y por qué vale la pena, si es que todavía es el caso, construir un nuevo proyecto de emancipación.

Lo que aporta no tanto el concepto como la práctica de la construcción de situaciones es la posibilidad de empezar a vislumbrar, de forma todavía muy precaria, una forma de transformación social que no se conforme con sólo mirar al pasado para poder explicar el presente. Si la segunda época de la I.S., sus textos de análisis más específicamente marxianos, suenan hoy demasiado lejanos es porque están demasiado atados a un contexto que ya no es el nuestro.

De un modo más preciso, la construcción de situaciones arrojan dos elementos que tendrán que ser claves en las próximas teorías y prácticas de transformación social radical: la primera es el principio de que no existe transformación social sin la transformación de la vida cotidiana. Este principio significa que toda acción política, ya sea revolucionaria o democráticoliberal, no será verdaderamente transformadora si no cambia el conjunto de condiciones materiales y vitales

\footnotetext{
Sobre el actual auge de una mitificación de la clase obrera, cf. O. Jones, Chavs. The Demonization of the Working Class, London, Verso, 2016; R. Romero Laullón (Nega); A. Tirado, La clase obrera no va al paraíso. Crónica de una desaparición forzada, Madrid, Akal, 2016.
} 
de los individuos. Aquí no se incluye solamente el trabajo asalariado, cuyas críticas cada vez son más numerosas $^{33}$, sino las formas de experimentar la propia cotidianeidad y todo lo que se incluye en ella, desde las formas de vida más íntimas a las más comunitarias.

El segundo elemento clave es que toda transformación social radical es un proceso que, por definición, no puede tener fin. Si la construcción de situaciones enseña que toda vida liberada no es más que un momento de ella, con su principio y su final, las nuevas formas de concebir la transformación social en las próximas décadas tendrán que eliminar conscientemente aquella idea del marxismo como una estación de llegada, una sociedad completamente liberada que espera al final de la cadena de las contradicciones sociales. No existe tal horizonte porque éste se diluye en la cotidianeidad.

Pese a que este principio pueda recordar a otras formulaciones del pasado, como pudieran ser las de "revolución permanente" de Trotsky, la enseñanza que arroja la construcción de situaciones en este sentido es que la transformación social no ocurre, o no solamente, a nivel institucional, al nivel de las diversas formas en las que operan los diferentes poderes sociales; la transformación social ha de desarrollarse, necesariamente, al nivel de las relaciones sociales más inmediatas, no sólo en el lugar de trabajo sino en la familia, en la sexualidad, en el ocio, en las rutinas, etc.

Dicho de otro modo, este segundo elemento que arroja la construcción de situaciones es el desplazamiento de la transformación social de lo institucional, entendido como el conjunto de organismos de poder que conforman una sociedad determinada, a lo relacional, en lo cual la institución tiene un papel evidentemente determinante pero que no reduce el conjunto de relaciones sociales que se dan en su interior.

En definitiva, la construcción de situaciones enseña que puede darse un cambio aparente en las estructuras del Estado, de la producción y del mercado (los ámbitos en los que gran parte de las teorías sociales han centrado sus esfuerzos) sin que la cotidianeidad más inmediata e íntima se vea realmente transformada. Es a partir de estos dos elementos, todavía muy precarios, que tenemos que empezar a pensar en cómo podrá funcionar la transformación social radical en las próximas décadas.

\section{Bibliografía}

Blanchard, D., La crisis de palabras, Madrid, Acuarela, 2007.

Debord, G., Correspondance, Vol. 1, Paris, Fayard, 1999.

-, Oeuvres, Paris, Gallimard, 2006.

Frayne, D., El rechazo del trabajo. Teoría y práctica de la resistencia al trabajo, Madrid, Akal, 2017.

Goldstone, J.A., "Rethinking Revolutions: Integrating Origins, Processes, and Outcomes", Comparative Studies of South Asia, Africa and the Middle East, 29, 1, 2009, pp. 18-32.

Hammond, J. L., "The Significance of Space in Occuppy Wall Street", Interface: A Journal For and About Social Movements, 5 , 2, 2013, pp. 499-524.

Hastings-King, S., "L'Internationale Situationniste, Socialisme ou Barbarie, and the Crisis of the Marxist Imaginary", Substance, 90, 1999, pp. 26-54.

Hayes, A., "Three Situationists Walk into a Bar", Axon, 8, 2015. (http://www.axonjournal.com.au/issue-8-1/three-situationistswalk-bar. Consultado por última vez el 15.04.2019).

Heynen, H., "New Babylon: The Antinomies of Utopia", Assemblage, 29, 1996, pp. 24-39.

Jappe, A., Guy Debord, Barcelona, Anagrama, 1998.

Jones, O., Chavs. The Demonization of the Working Class, London, Verso, 2016.

Laval, Ch. y Dardot, P., Común. Ensayo sobre la revolución en el siglo XXI, Barcelona, Gedisa, 2015.

Lefebvre, H., Les temps méprises, Paris, Stock, 1975.

-, Critique de la vie quotidienne. Vol. 1: Introduction, Paris, L'Arche, 1958.

-, La producción del espacio, Madrid, Capitán Swing, 2013.

Mahon, A., Surrealismo, Eros y politica, 1938-1968, Madrid, Alianza, 2009.

Marx, K. y Engels, F., Marx und Engels Werke (MEW). Vol. 4, Berlin, Dietz Verlag, 1977.

-, Marx und Engels Gesamtausgabe (MEGA), I. Abteilung, Band 2, Berlin, Dietz Verlag, 2009.

Reichert, W. O., “Anarchism, Freedom, and Power”, Ethics, 79, 2, 1969, pp. 139-149.

Romero Laullón, R. (Nega) y Tirado, A., La clase obrera no va al paraíso. Crónica de una desaparición forzada, Madrid, Akal, 2016.

Ross, K., "Lefebvre on the Situationist: An Interview", October, 79, 1997, pp. 69-83.

Thompson, E.P., La formación histórica de la clase obrera 1. Inglaterra: 1780-1832, Barcelona, Laia, 1977.

Vienet, R., Enragés: y situacionistas en el movimiento de las ocupaciones, Madrid, Castellote, 1978.

Vaneigem, R., Tratado del saber vivir para el uso de las jóvenes generaciones, Barcelona, Anagrama, 2008.

\footnotetext{
Para dos ejemplos actuales de las investigaciones en torno al trabajo asalariado, cf. D. Frayne, El rechazo del trabajo. Teoría y práctica de la resistencia al trabajo, Madrid, Akal, 2017; K. Weeks, The Problem with Work. Feminism, Marxism, Antiwork Politics, and Postwork Imaginaries, Durham and London, Duke University Press, 2011.
} 
Varon, J., Bringing the War Home. The Weather Underground, the Red Army Faction, and Revolutionary Violence in the Sixties and Seventies, Berkley/Los Angeles/London, University of California Press, 2004.

VV.AA., Guy Debord. Un art de la guerre, Paris, Gallimard, 2013.

VV.AA., Internationale Situationniste, Paris, Fayard, 1997.

VV.AA., Socialisme ou Barbarie. Anthologie, La Bussière, Acratie, 2007.

Weeks, K., The Problem with Work. Feminism, Marxism, Antiwork Politics, and Postwork Imaginaries, Durham and London, Duke University Press, 2011.

Wigley, M., Constant's New Babylon: The Hyper-Architecture of Desire, Rotterdam, Witte de With, Center for Contemporary Art, 1998. 